Proceedings of the 2011 Winter Simulation Conference

S. Jain, R.R. Creasey, J. Himmelspach, K.P. White, and M. Fu, eds.

\title{
SIMULATION-BASED STUDY OF HEMATOLOGY OUTPATIENT CLINICS WITH FOCUS ON MODEL REUSABILITY
}

\author{
Navonil Mustafee \\ Michael D. Williams \\ School of Business and Economics \\ Swansea University \\ Swansea, Wales, SA2 8PP, UK
}

\author{
Fiona Hughes \\ Abertawe Bro Morgannwg \\ University Health Board \\ Singleton Hospital \\ Swansea, Wales, SA2 8QA, UK
}

\author{
Korina Katsaliaki \\ School of Economics and Business Administration \\ International Hellenic University \\ Thessaloniki, 57001, Greece
}

\begin{abstract}
Several factors are expected to significantly increase stakeholders' interest in healthcare simulation studies in the foreseeable future, e.g., the use of metrics for performance measurement, and increasing patients' expectations. To cater to this, several strategies may have to be implemented in concert, e.g., development of skilled manpower and engagement with academia. The focus of this paper is on one such strategy -model reusability. The paper reports on an ongoing study that investigates the outpatient capacity and demand for specialist hematology services. The primary objective of this study is to test strategies for service consolidation. Yet another objective is to model the simulation with the granularity that would enable the model to reused in similar operations context. The paper discusses the reusability aspect and presents an overview of the hematology OPD case study; since this is an ongoing study the results of the simulation are not presented in this paper.
\end{abstract}

\section{INTRODUCTION}

Computer simulations are generally used because they are cheaper than building (and discarding) real systems; they assist in the identification of problems in the underlying system and allow testing of different scenarios in an attempt to resolve them; allow faster than real-time experimentation; provide a means to depict the behavior of systems under development; involve lower costs compared to experimenting with real systems; facilitate the replication of experiments; and provide a safe environment for studying dangerous situations like combat scenarios, natural disasters and evacuation strategies (Brooks et al. 2001; Pidd 2004). There are several kinds of simulation techniques, for example, Discrete-Event Simulation (DES), Agent-Based Simulation and System Dynamics, that are frequently applied to a wide range of problem scenarios in application areas such as (the following references are review articles), manufacturing and business (Jahangirian et al. 2010), commerce and defense (Eldabi et al. 2008), transport and logistics (Manivannan 2007), construction (Oloufa 1993), healthcare (Brailsford et al. 2009; Katsaliaki and Mustafee 2010), supply chains (Kleijnen 2005), among others. The focus of the paper is on DES, and in the reminder of this paper, the terms DES and simulation are used synonymously, unless otherwise stated.

This paper reports on an ongoing simulation-based study of the specialist hematology services that are offered to outpatients through four hospitals in the Neath and Port Talbot area in Wales, UK. The study is being conducted with a local NHS health board (Abertawe Bro Morgannwg University Health Board, Wales). There are two objectives of this study: (a) to investigates the outpatient capacity and de- 


\section{Mustafee, Hughes, Katsaliaki and Williams}

mand for specialist hematology services and to experiment with strategies for service consolidation, and (b) to develop a simulation model that could be reused for modeling de-centralized consultant clinics, wherein the consultants belong to a centralized resource pool. Thus, it is expected that this research will contribute to both practice (objective a) and the advancement of simulation theory (objective b).

With regard to the objective of reusability (i.e., objective b), at the very outset we state that we do not make the claim that our model can be reused by simulation practitioners to represent clinics exhibiting the characteristics that are consistent with the problem scenario we are trying to model. Indeed, the time and effort required to convey the intricacies of a model through knowledge transfer between modelers can frequently justify the creation of a model from scratch (a practitioner's experience in this context is provided in Robinson et al. (2004)). Our objective is more to do with encouraging those engaged in modeling healthcare systems (individual modelers or those working closely with each other) to be guided by the motivation of model reuse; this would increase the employment of reusable model components in new simulations being developed by these modelers themselves, and would facilitate exploration of opportunities for such model reuse amongst modelers (including those belonging to separate development groups). Although the subject of simulation model reuse is a generic one, this research lays particular emphasis on healthcare since the authors contemplate a significant increase in the number of healthcare simulation studies in the near future (section 2 presents the argument for this), and model reuse is recognized by the authors to be one of the important factors that could contribute to the increased throughput of models. Finally, reusability has cost implications; a computer model that costs less to develop will generally increase the cost-effectiveness of the overarching simulation-based study, ceteris paribus.

The remainder of the paper is structured as follows. Section 2 presents the arguments underpinning the contemplated rise in the demand for healthcare simulation studies in the coming years. Section 3 follows on from this argument and presents the case for model reusability. Section 4 reviews literature on the application of DES with particular reference to the modeling of healthcare clinics. The hematology outpatients' case study and the simulation being developed is presented in Section 5. Section 6 is the concluding section of the paper. It highlights the contribution and draws the paper to a close.

\section{THE RISING COST OF HEALTHCARE IN THE PERIOD OF DECREASE IN PUBLIC SPENDING: OPPORTUNITIES FOR SIMULATION}

There are several factors contributing to the rising costs of healthcare, for example, ageing population, increase in life expectancy, innovations in treatment, general inflation, among others. In this section we refer to three studies, all pertaining to the healthcare insurance industry in the US, that provide an estimate of the percentage increase in healthcare spending in distinct categories. Thorpe et al. (2005) refer to two broad categories, namely, the rise in treated disease prevalence and the rise in spending for treated cases, that account for an estimated $63 \%$ and $37 \%$ growth (Thorpe 2005) in US private insurance spending between 1987 and 2002 respectively. The factors contributing to the rise in treated disease prevalence include, (a) rising population that accounts for the rise in the "population prevalence of disease" (e.g., obesity, stress, air pollution, smoking), (b) changing treatment thresholds that have led to the treatment of more patients with asymptomatic conditions, and (c) new technologies that have led to more patients being treated; On the other hand, the factors contributing to the rise in spending for treated cases are usually associated with new treatment procedures and innovations in pharmacologic treatment options (e.g., there has been a five folds increase in spending for the delivery of newborns between 1987 and 2002) (Thorpe 2005; Thorpe et al. 2005). In a study commissioned by America's Health Insurance Plans (AHIP) and conducted by PricewaterhouseCoopers (PWC 2008), an increase of approximately $6.1 \%$ in healthcare premiums in the US between 2006 and 2007 have been attributed to three broad categories of price increases, namely, the general inflation (accounting for $2.8 \%$ ), healthcare price increases above inflation $(1.6 \%)$ - this includes price increases owing to higher priced technologies, and increased utilization of healthcare services $(1.7 \%)$. The factors contributing to the increased utilization of health services include, the availability of new treatment, aging population, increase utilization resulting from lifestyle choices 


\section{Mustafee, Hughes, Katsaliaki and Williams}

(e.g., smoking, alcohol intake, poor nutrition), and more intensive diagnostic testing/defensive medicine for preventive intervention (PWC 2008).

It can be argued that the aforementioned factors that have been identified in literature as contributing to the rising cost of healthcare insurance in the US, may equally account for the rise in healthcare costs in several other counties. This may be especially true for countries that are technologically advanced and economically strong (as they usually have an increased access to new healthcare innovations) and those that demonstrate an increase in ageing demographics. In countries where healthcare is publicly-funded, the rising costs have traditionally meant a rise in the healthcare budgets in real terms (i.e., rise in spending over and above the rate of inflation). In the UK for example, spending in the publicly-funded National Health Service (NHS) had increased more than nine-folds in real terms between 1949/50 and 2006/07 (Thompson 2009). However, an increase in spending does not necessarily translate into delivery of better healthcare services to the citizens. Indeed, one of the key findings of the report by the UK Comptroller and Auditor General on NHS hospital productivity is that, although there had been a significant increase in the resources going into the NHS during the period 2000-2010, the total NHS productivity had actually decreased by an average of $0.2 \%$ per year, with productivity in hospitals falling by around $1.4 \%$ per year (National Audit Office 2010).

The ongoing global financial and economic crisis (2007-present) has had spending implications on governmental budgets for public resources in several countries. In keeping with the example of the UK NHS, the healthcare budget is slated to rise by only $0.1 \%$ in real-terms between $2011 / 12$ to 2014/15; furthermore, efficiency savings of £20billion a year will have to be found by 2014/15 (Martin 2010). The call for the $£ 20$ billion savings was first made in 2009 by the Chief Executive of the NHS in England Sir David Nicholson (Ball and Patrick 2009). Now referred to as the Nicholson Challenge, this continues to be among the top priorities of the government (Health Committee of the House of Commons 2011).

Thus, on the one hand, governments are faced with the increasing cost associated with delivering healthcare; and on the other hand, they are faced with the prospect of reducing the spending in citizencentric services (including healthcare). This apparent contradiction - "provide better service at a lesser cost" (also acknowledged by other authors, including, Jahangirian, Taylor, and Young (2010)) - which may last for years to come, therefore necessitates the careful use of the allocated healthcare budgets through better and more informed decision making.

Decision making via the use of modeling tools has been extensively used in many systems management (Royston 1999), for example, decision support systems and modeling tools have been widely used by governments to get advice on making the best possible use of the allocation of public funds. They provide an insight into the working of a system and can be used to predict the outcome of a change in strategy. This is particularly useful when the system is very complex and when experimentation with the real systems is risky and or costly, both of which are true in the case of health services. With the increased use and capacity of computer technology, modeling techniques have developed rapidly and there are now a large number of approaches available (Royston 1999). For example, analytical models have been developed with the use of Markov chains, linear and dynamic programming, and other optimization methods; simulation models are created using techniques such as DES and Agent-Based Simulation. These wide array of modeling techniques enable the policy makers, researchers, managers, practitioners, consultants and other stakeholders to take informed decisions on utilizing available resources for efficient healthcare operations management. Indeed, there is a growing body of research on the application of simulation in the context of healthcare (refer to section 4 for a review of DES literature focusing on healthcare clinics). The authors conjecture that, with the increasing costs of delivering healthcare and the present trend of decreasing governmental outlays for health, there will be a growing requirement for simulation-based studies in the healthcare sector. Since most of the simulation studies expend considerable time and resources in implementing computer models, the reusability of models is an important factor that needs to be considered. This is discussed next with reference to DES. 


\section{MODEL REUSE}

Simulation model reuse refers to the creation of new models using pre-existing modeling artifacts like portions of simulation code, existing simulation components and complete models, with the purpose of reducing the time and cost for model development (Pidd 2002; Robinson et al. 2004). In its simplest form we can consider model reuse by a single development group (or indeed an individual modeler) using Commercial Simulation Packages (CSPs) like Simul8 and Anylogic (this is shown by the "bottom left" element of the Model Reusability Complexity Matrix in Figure 1). An extension of model reusability is the concept of separate development and user groups, whereby models are developed and validated by one group and then used to specify simulations by another group (Bortscheller and Saulnier 1992). The multiple development groups are represented in Figure 1 by the "bottom right" element (when such groups predominantly use CSPs) and the "top right" element (when specialized computing technologies are used - this is discussed in the next paragraph). With relation to the modeling costs, the potential for realizing economies of scale may increase in the multiple development group scenario.

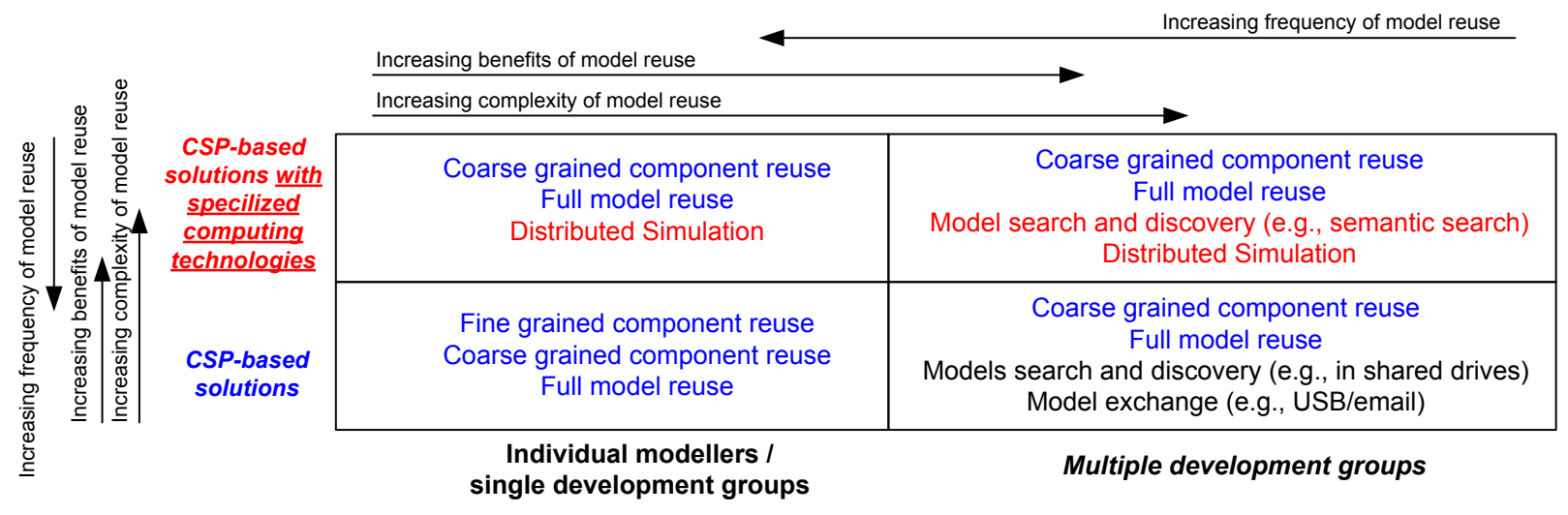

Figure 1: Model Reusability Complexity Matrix (adapted from Pidd 2002)

In a networked environment, simulation model reuse will generally involve searching and downloading model components for model building (Robinson 2005); refer to Bell et al. (2008) for an example of a CSP-based "semantic model discovery and import" approach to model reuse. Another technology that facilitates model reusability and interoperability in a networked environment is distributed simulation; refer to Mustafee et al. (2009) for a case study in CSP-based distributed simulation in healthcare. These specialized computing technologies can be used (together with CSPs) for model reusability by both the single development groups/single modelers (shown by the "top left" element in Figure 1) and the multiple development groups (shown by the "top right" element in Figure 1). However, both these approaches are highly specialized and require significant knowledge of computer programming and other computing concepts (e.g., ontology, web services, distributed systems), and the vast majority of simulation practitioners in industry are experts in M\&S, but not necessarily IT. As such, the practice of simulation in industry usually takes the form of simulation end-users relying on CSPs. In acknowledgement of these arguments, the focus of this research is on reusability that could be achieved exclusively through use of CSPs (refer to Paul and Taylor (2002) for a discussion on CSP-based model reuse); Further, our attempt is to reach out to the individual modeler (or the modelers working closely in a single development group) with the call for model reuse (shown by the "bottom left" element in Figure 1 above); we hope that this would, in time, facilitate exploration of opportunities for such model reuse amongst modelers in multiple development groups (shown by the "bottom right"). Finally, in Figure 1 above, we borrow the "complexity of implementation" and the "frequency of model reuse" indicators from Pidd (2002), and include our own indicator for "expected benefit of model reuse" (the direction of the arrows denote increasing complexity, increasing frequency, and increasing benefits of model reuse respectively). 
We now emphasize the importance of reusability in healthcare M\&S in relation to the 2-by-2 Matrix Framework for Modeling and Simulation Cost-Effectiveness that has been proposed by Jahangirian, Taylor, and Young (2010). In doing so, we focus on the two primary advantages that may be derived through use of reusable models, namely, (a) an increase in model throughput (brought about by a decrease in model development time), and (b) reduction in the cost of model development. Centralized healthcare systems (such as the NHS) have opportunities to benefit from Economies of Scale (Jahangirian, Taylor, and Young 2010), and an increase in the number of models being developed for use in such systems will generally contribute towards increasing the economies of scale. This will lead to higher benefits being derived ("top left" and "top right" elements of the 2-by-2 matrix - see Figure 2 [left]). Next, a decrease in the total effort in developing the computer model will generally be reflected in the reduction in the cost of conducting a simulation-study. This is shown in the "top left" and the "bottom left" elements of the 2-by2 matrix (see Figure 2 [left]). Looking at the advantages of model reuse in concert (with reference to the 2-by-2 matrix, this means overlaying the "top left" and the "top right" elements [signifying an increase in benefit] over the "top left" and the "bottom left" elements [signifying a cost reduction]), we identify the 2-by-2 matrix element ("top left") associated with high benefit and low cost. Thus, model reuse can decrease the costs associated with conducting simulation studies and, at the same time, can potentially increase the benefits derived through its use. In the 2-by-2 matrix framework, the high benefit and low cost segment is associated with cost-effectiveness. It therefore follows that the simulation-based studies that have a focus on model reusability will generally be more cost-effective. Jahangirian, Taylor, and Young (2010) have also used the 2-by-2 matrix to chart the cost-effectiveness of two groups of M\&S techniques, namely, simulation and mathematical modeling. Figure 2 [right] borrows from this and shows the original ellipse representing "simulation" in the cost-effectiveness matrix, and indicates its probable shift in terms of benefit and cost (represented by dotted ellipse) in the event of model reusability. Thus, Figure 2 [right] shows that simulation studies that focus on model usability will have the potential at realizing higher benefits and at a lesser cost.

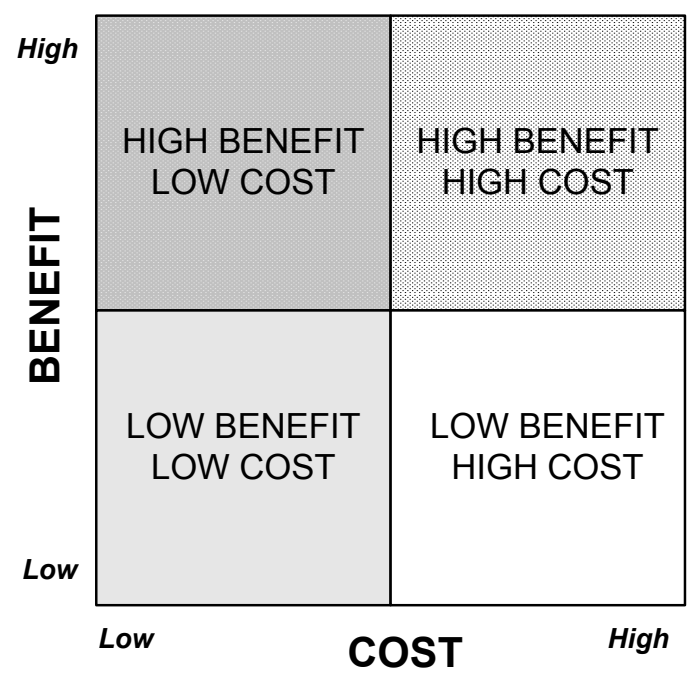

Higher benefit derived from an increase in model throughput, brought about by model reuse

Decrease in the cost of conducting simulation studies, brought about by model reuse

Model reuse can lead to higher benefits (e.g., economies of scale) being derived at lower costs

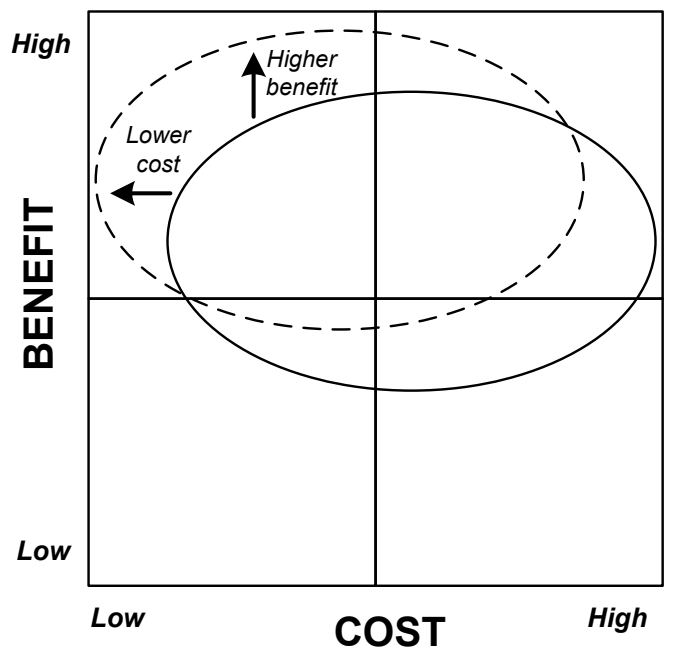

Ellipse showing the original cost-effectiveness of the simulation category, as depicted in Jahangirian, Taylor, and Young (2010) $\uparrow \leftarrow \begin{aligned} & \text { Shifts in benefit and cost brought about through model } \\ & \text { reuse }\end{aligned}$

Ellipse showing the increased cost-effectiveness of - _ the simulation category, brought about by model reuse

Figure 2: The Matrix Framework (Jahangirian, Taylor, and Young 2010) identifying opportunities for deriving higher benefits (left) and an increased cost-effectiveness (right) brought about through model reuse. 


\section{MODELLING OF HEALTHCARE CLINICS: A LITERATURE REVIEW}

Improving patients' flow in healthcare clinics has been the focus of many studies. Modeling methodologies that have been applied in these studies include, heuristics, linear programming, Markov chains, queuing theory and simulation. Especially, computer simulation has been extensively used to model healthcare clinics over the past 30 years and more. The most commonly used simulation techniques for this purpose is system dynamics and DES, with the latter significantly outnumbering the former. DES has been used to model both single-facility and multi-facility healthcare clinics such as, outpatient clinics, emergency departments, surgical centers, orthopedic departments, and pharmacies. They deal with issues such as, patient scheduling and admissions, patient routing and flow schemes, staff scheduling, work flow, allocation of resources when sizing and planning beds, rooms, and staff personnel (Jun, Jacobson, and Swisher 1999). An excellent comprehensive survey on the application of discrete event simulation in healthcare clinics over the $80 \mathrm{~s}$ and $90 \mathrm{~s}$ is presented in Jun et al. (1999). In their survey the authors refer to over 100 articles published in this area.

Over the last decade the work on healthcare clinics has continued to flourish. Some of these studies are presented here (starting from the most recent). Most of the research in this field experiments with simulation models of clinics with variable patients arrival patterns such as outpatient clinic settings and surgical care clinics. The focus is on scheduling patients appointments, predicting patient no-shows and reducing patient waiting time (Rohleder et al. 2010; Lee and Yih 2010; Glowacka, Henry, and May 2009; Vasilakis et al. 2007, Giachetti et al. 2005; Harper and Gamlin 2003). They try to develop scheduling rules for different patients and referral types, and test alternative staff utilization methods and care teams configurations for scheduling patients appointments more efficiently and improving clinic performance. Taylor and Kuljis (1998) and Paul (1995) present an overview of how DES can be used to investigate the reduction of waiting times in an outpatient clinic by using commercial simulation packages.

There are several examples of simulation studies that have focused on increasing the performance of clinics with less variable patients arrival patterns. For example, the aim of the study by Berg et al. (2010) was to evaluate the allocation of resources for optimal use of facilities for colonoscopy screening. Operational configurations were compared by varying the number of endoscopists, procedure rooms, the patient arrival times, and procedure room turnaround time. Performance measures included the number of patients served during the operating hours of the clinic and utilization of key resources. In the same spirit, Villamizar et al. (2011) developed a computer model to analyze the performance of a standard physiotherapy clinic and experimented with patients numbers, resources available at the clinic and patient arrival schedules. Alexopoulos et al. (2001) developed a low cost simulation model populated by a workflow observation Excel spreadsheet that could be completed by clinic staff themselves. The model was tested on childhood immunization delivery services. Some other studies, for example, Washington (2010) and Aaby et al. (2006) have built DES models that could be used to improve clinic planning for dispensing medications and vaccines during disease outbreaks and to estimate the throughput of the vaccination clinic as the number of clients increased and as staff members were reassigned to different workflows.

Finally, we refer to the study on the "operational functioning of a family practice within a physician partnership network " by Swisher et al. (2001) and draw some parallels with our hematology OPD case study. The model developed by Swisher et al. (2001) captured both the operations of the physician clinic and the centralized information center. The purpose of the information store was to manage all nonmedical operations, e.g., patient scheduling and billing, so that the individual member clinics could benefit from centrally managed operations with the overall goal to increase patient flow and customer satisfaction. In the concluding section of their paper the authors state that an area of future research could be to expand the single member clinic model to multiple member clinic model and to study its effects upon the centralized information centre. This statement of future research by Swisher et al. (2001) could be considered as the starting point of our hematology OPD study, as we model multiple clinics that have an overarching centralized structure, albeit to test strategies for service consolidation. 


\section{HEMATOLOGY OPD CASE STUDY AND THE SIMULATION MODEL}

Abertawe Bro Morgannwg (ABM) University Health Board (UHB) is amongst the largest health boards in Wales. It has around 17,000 staff serving a population of approximately 600,000 and covering the areas of Bridgend, Neath Port Talbot and Swansea (ABMUHB 2011). It operates a total of 16 hospitals, with four of them having 24-hour A\&E - Morriston Hospital (MH), Princess of Wales Hospital (PWH), Neath Port Talbot Hospital (NPTH) and Singleton Hospital (SH). The catchment area of ABMUHB is logically divided into the West zone (which comprises of $\mathrm{MH}$ and $\mathrm{SH}$ ) and the East zone (PWH and NPTH). SH houses the Cancer Centre and the Department of Hematology and it serves the entire ABMUHB catchment area. The Department of Hematology has a centralized resource pool consisting of seven consultant hematologists. These consultants are responsible for outpatients, day patients and inpatients in all the four hospitals (these hospitals, in turn, receive referrals from the other ABMUHB hospitals and the GP clinics).

In the present structure of delivery of hematology OPD services, outpatient clinics are held in all the four hospitals. However, a study conducted by ABMUHB and the Royal College of Pathologists recommended a proposed reorganization through consolidation of outpatient clinics from four hospitals to two. It proposed that the clinics are to be based in SH (catering mainly to the West Zone) and PWH (catering mainly to the East Zone). The recommendations looked into the efficiency aspect of the operation. However, it did not take into account the cost implications. Furthermore, a consolidation of outpatient clinics will also have to take into consideration the distance that has to be travelled by the patients to attend a clinic, the preferences of patients (some may want to travel locally), the funding available, etc. Finally, overhauling the delivery structure would require many operational changes to be made (e.g., alterations in the existing appointment system, changes in clinic schedule, reorganization of resources) and this involves an element of risk. A simulation-based study can mitigate some of these risks as it allows for experimenting "what-if?" scenarios prior to implementing the changes in the real system.

The study presented in this paper focuses on the capacity and demand for hematology OPD across SH, MH, NPTH and PWH. The objectives of this study are as follows: (a) to test strategies for service consolidation (this is the main objective); (b) experimenting with policies that could reduce the Referral To Treatment (RTT) waiting time (presently 26 weeks with first appointment at 18 weeks; ABMUHB cancer services have a goal of reducing this to 17 weeks with first appointment at 10 weeks); (c) experimenting with the provision for specialist clinics (for specific blood disorders); and (d) analyzing the effects of appointment cancellation and rescheduling on the waiting list.

Figure 3 depicts the flow of patients in the system. There are usually two ways in which patients can enter the system - (a) New cases are referred to the First Appointment Team (FAT) - there are presently three such teams for SH, MH and NPTH/POW and each of them maintain a separate waiting list; (b) For Urgent Suspected Cancer (USC) cases, referrals are usually sent through to one of the two "Safe Heaven" fax machines (one each in East zone and West zone). In case of (a), the referrals are sent to the hematology consultants for prioritization into urgent slots (first appointment in 2-4 weeks) and routine slots (patients to be seen within 18 weeks). In case of (b), it is essential that the patients are seen as soon as possible and they are immediately allotted the 10-day appointment slot. Letters are then sent to the patients informing them of New Patient (NP) appointment and the clinics that they have been allocated to.

The 10-day slots and the urgent slots are non pre-booked slots, thus, the patients are given appointments without previous consultation on their availability; the routine slots allows for time to consult and agree on an appointment date and time with the patients (pre-booked slots). This broad categorization of appointments into pre-booked and the non pre-booked slots may be important when analyzing data pertaining to patients that Did Not Attend (DNA - no shows) or were Unable To Attend (UTA - patients that informed the clinics that they will not be able to keep an appointment). For both DNAs and UTAs the patients are usually given another NP appointment, however, the RTT clock is stopped until the time the patients have shown up for their new appointments.

A NP appointment usually leads to a Follow-Up Appointment (FUP). Only in a minority of cases are the patients discharged subsequent to the first appointment. The reason for this is, in the specialist area of 


\section{Mustafee, Hughes, Katsaliaki and Williams}

hematology diagnosis is usually only possible subsequent to the consultants having looked at patient blood reports. Thus, in the course of a NP appointment the hematologists usually order for certain blood tests; in the FUP appointments the consultants refer to these blood reports and this enables them to make a diagnosis (at this point the RTT clock is stopped). It is to be noted here that, for some patients at least, there may be an opportunity to complete a diagnosis in the course of the first NP appointment. This may be possible since the consultant hematologists would already have accessed the GP referrals and the patient records (on the basis of which patients were earlier prioritized and allocated to either urgent slots or regular slots), and this might present them an opportunity to order the blood tests prior to the first patient consultation. The forms for the blood test can then be included together with the NP appointment letters and the patients may be requested to provide blood for testing prior to the first appointment (thus ensuring the availability of the blood reports during the first appointment). This can significantly reduce the RTT waiting time, and it is among the strategies to be experimented with the simulation model that be being developed.

Subsequent to patients having been seen by the consultants (either as a NP or a FUP), a clinical decision has to be made as to whether further FUP appointments are required (some patients may be present in the system for several years, e.g., wait and watch patients). There are two options for scheduling FUP appointments - (a) FUP appointments required within 6 weeks are usually given in the clinics; (b) FUP appointment requests exceeding 6 weeks are placed in the waiting list (referred to as the FUP Knock-Up List). These are two such lists at present, one each for the West and the East zones respectively.

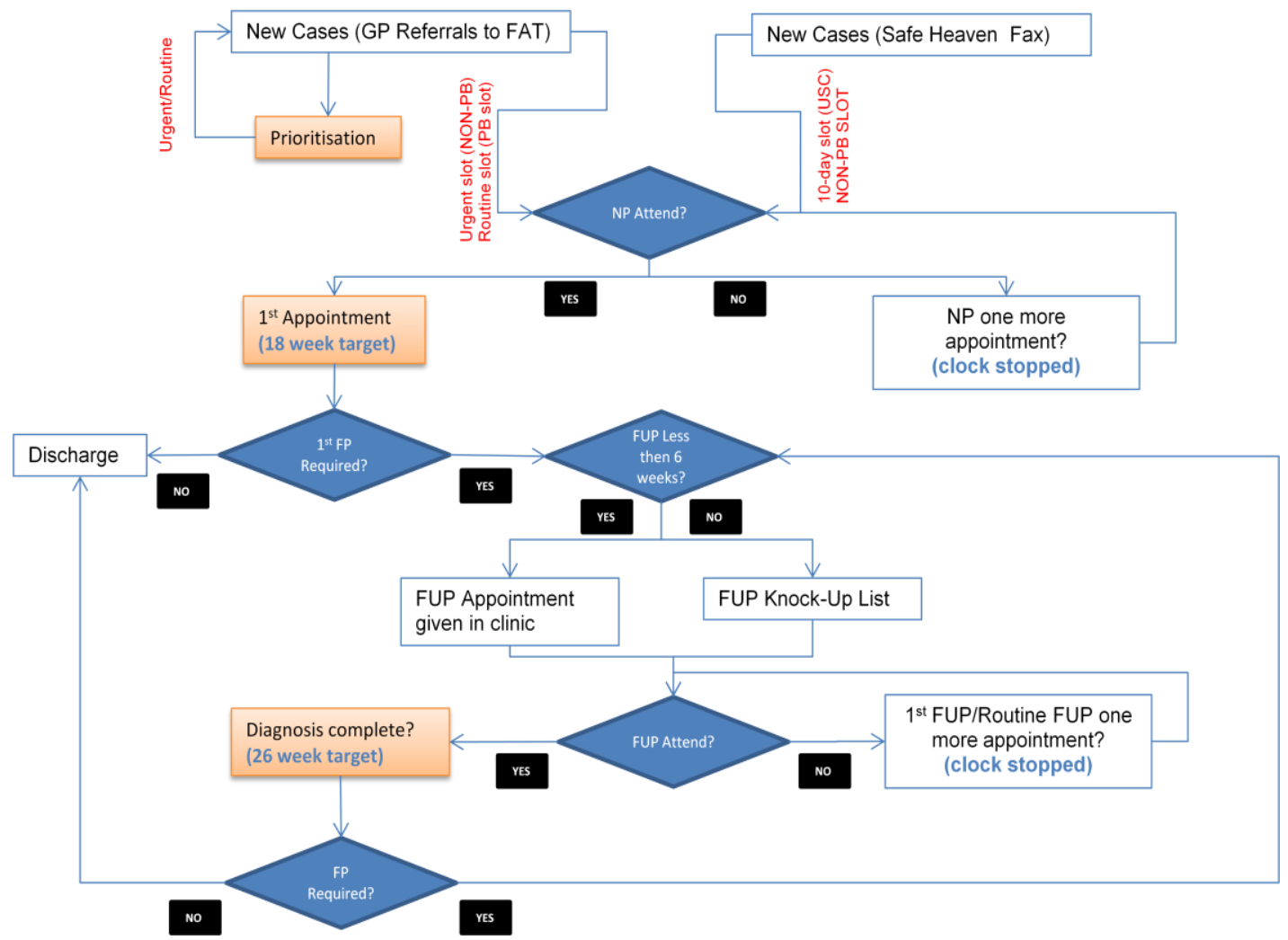

Figure 3: Flowchart of the hematology OPD patient pathway

As has been noted earlier, the central pool of hematologists can be allocated to clinics in any four of the hospitals. Most of the clinics run weekly at a predefined day of the week and time (there are two clinics that take place in alternative weeks). Each clinic has a fixed number of appointment slots, e.g., clinic $X$ may have $5 \mathrm{NP}$ appointment slots (comprising of, for example, two 10-day slots, one urgent slot, and the 
two regular slots) and 7 FUP slots. Also, some clinics may have several consultants assigned to them, for example, an afternoon clinic in SH has three hematologists, who between them, are responsible for a total of 3 NP and 56 FUP. Figure 4 presents the screenshot of the Simul8 model that is being developed. It models all the aforementioned characteristics of the system under scrutiny, for example, patients, patient categories, individual consultants, pools of consultants, nine clinics operating in four hospitals, first appointment and FUP appointments.

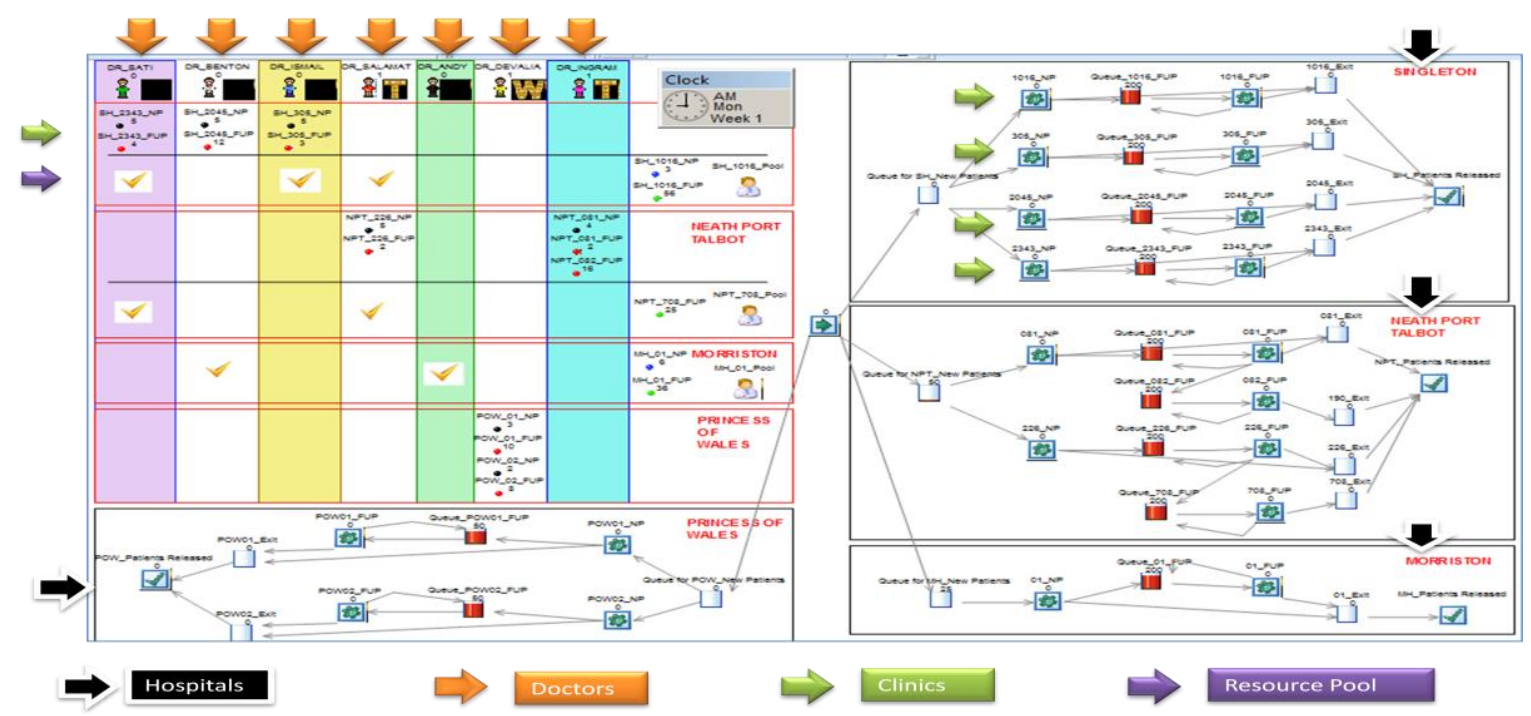

Figure 4: The hematology OPD model being developed using DES simulation package Simul8

One of the objectives of this study is to implement the model with the granularity that would enable it to be tested for suitability in similar healthcare operations contexts, namely, centralized hospital departments providing specialist outpatients services through an appointment-based de-centralized delivery structure. The focus on reusability has necessitated certain trade-offs to be made. Thus, although the simulation models the centralized resource pool comprising of the consultant hematologists, it does not model the local resources like the nurses and the receptionists (although they are essential for efficient functioning of the system). Further, the OPD model does not include the processes associated with the blood tests (although, in some cases, the FUP patients may be sent for blood tests and asked to return in a couple of hours to the same clinic); including this would have compromised the reusability objective to an extent, since not all specialist treatments have a requirement for additional processes (and, indeed, some may have more than one such process). Next, the operating hours of the clinics are fixed, and the service time for the patients (consultation time) are calculated based on the number of NP and FUP slots available in each clinic. Although this strategy would not adequately capture values for variables like patient waiting time, patient service time, utilization rate of the clinics (most clinics run past their scheduled closing time), etc., it is considered adequate for modeling a system that is based on appointments (this also means that our model is unsuitable for modeling A\&E departments). Also, the only bottlenecks in the system are the pre-defined numbers of NP and FUP appointment slots, and the pool of consultant hematologists. Finally, it is to be noted that this is an on-going study and we are further developing the model with inputs from the stakeholders. We expect to present the results of the simulation experiments in a subsequent publication.

\section{CONCLUSION}

A simulation-based study usually involves several steps, e.g., planning the study, problem formulation, data collection, conceptual model definition, model development (including verification and validation) 


\section{Mustafee, Hughes, Katsaliaki and Williams}

and experimentation (Law and Kelton 2000), and is generally time consuming; It also has a cost element, e.g., purchase and maintenance of hardware and simulation software, training costs, costs incurred towards person time (for modeling, data collection, experimentation, etc.) and consultancy fee (Robinson 2004) - also, the cost element includes the opportunity costs for the stakeholders. Reusing simulation models can potentially reduce the time and costs associated with model development. In this paper the authors argue that the rising cost of healthcare, coupled with decreasing healthcare budgets and increasing end-user expectations, will stimulate further interest for simulation-based studies amongst healthcare stakeholders. To cater to this expected increase in demand for healthcare models, it is important to consider the model reusability aspect. The authors conjecture that, the subject area of healthcare simulation, in particular its application in the context of operations management, has reached a level of maturity that warrants the principles of model reuse be incorporated in the model development process right from the outset. The authors then present an overview of the hematology OPD case study that focuses not only on meeting the stakeholders' objectives but also on model reusability.

\section{REFERENCES}

Aaby, K., J. W. Herrmann, C. S. Jordan, M. Treadwell, and K. Wood. 2006. "Montgomery Countys Public Health Service uses operations research to plan emergency mass dispensing and vaccination clinics." Interfaces 36(6):569-579.

ABMUHB. 2011. "ABM University Health Board (UHB) - About Us". Accessed April 7, 2011. http://www.wales.nhs.uk/sitesplus/863/page/41169.

Alexopoulos, C., D. Goldsman, J. Fontanesi. M. Sawyer, M. D. Guire, D. Kopald, and K. Holcomb. 2001. "A discrete-event simulation application for clinics serving the poor." In Proceedings of the 2001 Winter Simulation Conference, Edited by B. A. Peters, J. S. Smith, D. J. Medeiros, and M. W. Rohrer, 1386-1391. Washington, DC, USA: IEEE Computer Society.

Ball, J., and S. Patrick. 2009. "NHS chief tells trusts to make £20bn savings," The Telegraph (13 Jun 2009). Accessed February 22, 2011. http://www.telegraph.co.uk/health/healthnews/5524693/NHSchief-tells-trusts-to-make-20bn-savings.html.

Bell, D., N. Mustafee, S. de-Cesare, M. Lycett, S. J. E. Taylor, and P. A. Fishwick. 2008. "Ontology Engineering for Simulation Component Reuse." International Journal of Enterprise Information Systems 4(4): 47-61.

Berg, B. B. Denton, H. Nelson, H. Balasubramanian, A. Rahman, A. Bailey, and K. Lindor. 2010. "A Discrete Event Simulation Model to Evaluate Operational Performance of a Colonoscopy Suite." Medical Decision Making 30(3):380-387.

Bortscheller, B. J., and E. T. Saulnier. 1992. "Model reusability in a graphical simulation package." In Proceedings of the 1992 Winter Simulation Conference, edited by B.L. Nelson, W.D. Kelton, and G.M. Clark, 764-772. Piscataway, NJ: Institute of Electrical and Electronic Engineers Inc.

Brailsford, S. C., P. R. Harper, B. Patel, and M. Pitt. 2009. "An analysis of the academic literature on simulation and modeling in health care." Journal of Simulation 3(3):130-140.

Brooks, R., S. Robinson, and C. Lewis. 2001. Simulation and inventory control. Operational Research Series. Hampshire, UK: Palgrave.

Eldabi, T., M. Jahangirian, A. Naseer, L. Stergioulas, T. Young, and N. Mustafee. 2008. "Applications of simulation techniques in commerce and defence: A systematic survey." In Proceedings of the OR Society 4th Simulation Workshop (SWO8), pp. 275-284.

Giachetti, R., E. Centeno, M. Centeno and R. Sundaram. 2005. "Assessing the viability of an open access policy in an outpatient clinic: A discrete-event and continuous simulation modeling approach." In Proceedings of the 2005 Winter Simulation Conference, edited by M. Kuhl, N. Steiger, F. Armstrong, and J. Joines, 2246-2255. Piscataway, NJ: Institute of Electrical and Electronic Engineers Inc.

Glowacka, K, R. Henry, and J. May. 2009. "A hybrid data mining/simulation approach for modelling outpatient no-shows in clinic scheduling." Journal of the Operational Research Society 60(8):1056-1068. 


\section{Mustafee, Hughes, Katsaliaki and Williams}

Harper, P. R. and H. M. Gamlin. 2003. Reduced Outpatient Waiting Times with Improved Appointment Scheduling: A Simulation Modelling Approach. OR Spectrum 25(2):207-222.

Health Committee of the House of Commons. 2011. Third Report on NHS Commissioning (published 13 January 2011). Accessed 2011. http://www.publications.parliament.uk/pa/cm201011/cmselect/cmhealth/513/51302.htm.

Jahangirian, M., S. J. E. Taylor, and T. Young. 2010. "Economics of Modeling and Simulation: Reflections and Implications for Healthcare." In Proceedings of the 2010 Winter Simulation Conference, edited by B. Johansson, S. Jain, J. Montoya-Torres, J. Hugan, and E. Yücesan, 2283-2292. Piscataway, NJ: Institute of Electrical and Electronic Engineers Inc.

Jun, J., S. Jacobson, and J. Swisher. 1999. "Application of discrete-event simulation in health care clinics: A survey." Journal of the Operational Research Society 50(2):109-123.

Katsaliaki, K. and N. Mustafee. 2010. "Applications of Simulation Research within the Healthcare Context." Journal of the Operation Research Society, advance online publication, October 13, 2010. DOI:10.1057/jors.2010.20.

Kleijnen, J. P. C. 2005. "Supply chain simulation tools and techniques: a survey." International Journal of Simulation and Process Modelling 1(1-2):82-89.

Law, A. and W. Kelton. 2000. Simulation Modeling and Analysis, 3rd edition. New York: McGraw-Hill.

Lee, S., and Y. Yih. 2010. "Analysis of an Open Access Scheduling System in Outpatient Clinics: A Simulation Study". Simulation: Transactions of the Society for Modelling and Simulation 86(8-9):503518.

Manivannan, M. S. 2007. "Simulation of Logistics and Transportation Systems." In Handbook of Simulation: Principles, Methodology, Advances, Applications, and Practice, Edited by J. Banks, Chapter 16. Hoboken, NJ, USA: John Wiley \& Sons.

Martin, D. 2010. "Spending review: NHS protected but must save $£ 20 b$," MailOnline article (1 October 2010). Accesed April 2, 2011. http://www.dailymail.co.uk/news/article-1322252.

Mustafee, N., S. J. E. Taylor, K. Katsaliaki, and S. Brailsford. 2009. "Facilitating the Analysis of a UK National Blood Service Supply Chain Using Distributed Simulation." Simulation: Transactions of the Society for Modelling and Simulation 85(2): 113-128.

National Audit Office. 2010. "Management of NHS hospital productivity," Report by the Comptroller and Auditor General (HC 491). Accessed April 2, 2011. http://www.nao.org.uk/publications/1011/nhs_hospital_productivity.aspx.

Paul, R. J. 1995. "Outpatient clinics: the CLINSIM simulation package." OR Insight 8(2):24-27.

Paul, R. J., and S. J. E. Taylor. 2002. "What use is model reuse: is there a crook at the end of the rainbow?" In Proceedings of the 2002 Winter Simulation Conference, Edited by E. Yücesan, C. H. Chen, J. L. Snowdon, and J. M. Charnes, pp. 648-652. Piscataway, NJ: Institute of Electrical and Electronic Engineers Inc.

Pidd, M. 2002. "Simulation software and model reuse: a polemic." In Proceedings of the 2002 Winter Simulation Conference, edited by E. Yücesan, C. H. Chen, J. L. Snowdon, and J. M. Charnes, pp. 772775. Piscataway, NJ: Institute of Electrical and Electronic Engineers Inc.

Pidd, M. 2004. Computer simulation in management science, 5th edition. Chichester, UK: John Wiley.

Jahangirian, M., T. Eldabi, A. Naseer, L. K. Stergioulas, and T. Young. 2010. "Simulation in manufacturing and business: A review.” European Journal of Operational Research 203(1): 1-13.

Robinson, S. 2004. Simulation: The Practice of Model Development and Use. Chichester, UK: John Wiley.

Robinson, S. 2005. "Distributed simulation and simulation practice." Simulation 81(5): 5-13.

Robinson, S., R. E. Nance, R. J. Paul, M. Pidd, and S. J. E. Taylor. 2004. "Simulation model reuse: definitions, benefits and obstacles." Simulation Modelling Practice and Theory 12(7-8):479-494.

Rohleder, T., T. Huschka, J. Egginton, D. O'Neil, and N. Woychick. 2010. "Modeling Care Teams at Mayo Clinic." In Proceedings of the 2010 Winter Simulation Conference, edited by B. Johansson, S. 
Jain, J. Montoya-Torres, J. Hugan, and E. Yücesan, 2304-2314. Piscataway, NJ: Institute of Electrical and Electronic Engineers Inc.

Royston, G. 1999, “Commentary: trials versus models in appraising screening programmes." British Medical Journal 318(7180): 360-361.

Swisher J. R., S. H. Jacobson, J. B. Jun, and O. Balci. 2001. "Modeling and analyzing a physician clinic environment using discrete-event (visual) simulation." Computers \& Operations Research 28(2):105-125.

Taylor, S. J. E., and J. Kuljis. 1998. "Simulation in health care management: modelling an outpatient clinic." OR Insight 11(3):7-11.

Thompson, G. 2009. "NHS Expenditure in England," Standard Note SN/SG/724 (2 June 2009). House of Commons Library. Accessed April 2, 2011. http://www.nhshistory.com/parlymoneypapter.pdf.

Thorpe, K. E. 2005. "The Rise In Health Care Spending And What To Do About It." Health Affairs 24(6): 1436-1445.

Thorpe, K. E., C. S. Florence, D. H. Howard, and P. Joski. 2005. "The Rising Prevalence Of Treated Disease: Effects On Private Health Insurance." Health Affairs 24(2005): w317-w325.

Vasilakis, C., B. G. Sobolev, L. Kuramoto, and A. R. Levy. 2007. "A simulation study of scheduling clinic appointments in surgical care: individual surgeon versus pooled lists." Journal of the Operational Research Society 58(2):202-211.

Villamizar, J. R., F. C. Coelli, W. C. A. Pereira, and R. M. V. R. Almeida. 2011. "Discrete-event computer simulation methods in the optimisation of a physiotherapy clinic." Physiotherapy 97(1):71-77.

Washington, M. L. 2009. "Evaluating the Capability and Cost of a Mass Influenza and Pneumococcal Vaccination Clinic via Computer Simulation." Medical Decision Making 29(4):414-423.

\section{AUTHOR BIOGRAPHIES}

NAVONIL MUSTAFEE is a lecturer in Information Systems and Operations Management in the School of Business and Economics, Swansea University (UK). His research interests are in simulation methodologies, healthcare simulation, parallel and distributed simulation, technology adoption and diffusion, and grid computing. His e-mail address is n.mustafee@swansea.ac.uk.

FIONA HUGHES is a Deputy General Manager for Cancer Services Directorate within Abertawe Bro Morgannwg University Health Board. Having qualified as a Registered General Nurse in St Mary's Hospital, Paddington in 1991 she gained experience in a range of services including pre-operative assessment, surgical nursing and cancer care management. She completed her BSc in Nursing within Swansea University in July 2000 and then successfully gained her Masters in Business Administration in 2003. Her areas of interest include project management and management of change within an acute care setting. Her email address is fiona.hughes@wales.nhs.uk.

KORINA KATSALIAKI is a lecturer in Technology and Operations Management at the International Hellenic University (IHU), Greece, and a part-time academic member at the Open University of Greece and Cyprus. Her research interests include health service research, the modeling of supply chains, educational simulation games and simulation modeling methodologies. Her email address is k.katsaliaki@ihu.edu.gr.

MICHAEL D. WILLIAMS is a Professor in the School of Business and Economics at Swansea University in the UK. He holds a BSc from the CNAA, an MEd from the University of Cambridge, and a PhD from the University of Sheffield. He is the author of numerous refereed and invited papers, and has obtained research funding from sources including the European Union, the Nuffield Foundation, and the Welsh Assembly Government. He can be contacted at: m.d.williams@swansea.ac.uk. 